\title{
La población negra en la Colombia de hoy: dinámicas sociodemográficas, culturales y políticas
}

Olivier Barbary

Fernando Urrea

G ste número de la revista Estudos Afro-Asiáticos está dedicado a

L la población negra en la sociedad colombiana contemporánea y su dinámica sociodemográfica, política y cultural. Como resultado de un largo proceso histórico, las relaciones sociales en la sociedad colombiana, al igual que otras sociedades latinoamericanas, se encuadran en una jerarquía social racializada, la cual, a pesar de variaciones temporales y regionales, se concretiza casi sistemáticamente en fenómenos de discriminación socio-racial de distintos tipos: residencial, económico y social, cultural y político etc. El punto de vista común en los artículos aquí reunidos es una perspectiva de análisis de este componente racial de la organización social colombiana, tomando en cuenta nuevas lógicas étnicas de identificación y diferenciación de las poblaciones que el discurso multiculturalista dominante ha impuesto en Colombia, primero a través de la Constitución de 1991 y luego, en el caso particular de las poblaciones negras, a través de la Ley 70 de 1993, o Ley de negritudes. En tal sentido, una pregunta que tiene una importante repercusión política atraviesa los distintos artículos: ¿Como se relacionan la emergencia actual de nuevas identidad(es) afrocolombiana(s) y sus manifestaciones sociales, culturales y políticas (autopercepción individuales y colectivas, nuevas producciones cul- 
turales "negras", nuevas reivindicaciones y estructuraciones políticas) con las desigualdades y las discriminaciones socio-raciales, la lucha contra el racismo y el acceso a la ciudadanía? Desde esta perspectiva, pensamos que los cincos estudios presentados cuestionan, cada uno a su manera, el proyecto de sociedad multiculturalista desarrollado por la nueva constitución y la reelaboración del modelo de ciudadanía que conlleva.

Lo dicho anteriormente tiene que ver también con la evolución reciente de la producción científica y estadística sobre esta cuestión en Colombia. Hasta la década de los noventa, el debate académico y político sobre el tema tenia lugar en medio de una "invisibilidad histórica" de la población negra en Colombia que, a pesar de las denuncias y esfuerzos de los pioneros de los estudios afrocolombianos como de N. de Friedemann y J. Arocha, se extendía desde la esfera jurídica y política hacia la producción estadística nacional y local, y por consiguiente con pocas excepciones, a la investigación demográfica y sociológica. Indiscutiblemente, este contexto se ha modificado radicalmente en los diez últimos años, tanto a escala nacional, con la inclusión de preguntas de autoidentificación étnica en el censo de 1993 y por el color de piel en la Encuesta Nacional de Hogares de 2000, como a escala local con programas de investigación y encuestas específicas, de corte demográfico o socio-antropológico, especialmente en grandes ciudades (Cali, Bogota etc.). El fin de esta "invisibilidad”, más allá de su primer resultado -la estimación confiable de los pesos demográficos nacionales y locales de la población negra y mulata (como se verá en el segundo artículo, cerca de $20 \%$ de la población total del país) - tiene, como vamos a ver, impactos profundos sobre la estructuración teórica y metodológica del campo de los "estudios afrocolombianos", y más generalmente de las ciencias sociales en Colombia.

Con este dossier queremos, más que presentar la colección de una serie de artículos que no pueden pretender ser representativos de todos los estudios afrocolombianos, reunir contribuciones a un nuevo enfoque sobre las dinámicas de las relaciones socio-raciales en Colombia. En efecto los cinco textos mantienen sobre este tema un punto de vista socioantropológico y sociodemográfico compartido, aunque con métodos específicos según el tipo de artículo y la disciplina de cada investigador. Se trata de analizar los diversos componentes de esas dinámicas, a partir de una óptica contemporánea y contextualizada social y geográficamente de esta población, diferenciándose de los estudios clásicos afrocolombia- 
nos y otros más recientes que van en otra dirección teórica, muy diferente a la aquí presentada. Si pudiera darse una primera calificación de este enfoque es su particular énfasis al análisis de las situaciones o contextos nacionales, regionales y locales de la sociedad colombiana en las que está inserta la población negra, sin buscar un sentido esencialista o de orígenes que enfatiza más la tradición cultural como permanente en el tiempo. Por el contrario, los autores aquí reunidos resaltan los procesos de construcción y transformación que experimenta la población negra, la heterogeneidad de su composición socioeconómica y de sus prácticas culturales y políticas identitarias, en adecuación a las situaciones históricas existentes en una sociedad jerarquizada racialmente, y también a las variaciones regionales y locales que matizan el juego de las apariencias fenotípicas.

En segundo lugar, los artículos aquí reunidos tienen también una perspectiva común del análisis de los procesos raciales como componentes de la sociedad colombiana y por lo mismo como generadores de desigualdades sociales. El factor racial, aunque intercepta las dimensiones de clase, género y edad, e interactúa con esas dimensiones para producir discriminaciones socio-raciales, mantiene una especificidad en la producción de desigualdades sociales. Sin embargo, hay que advertir bien que las lógicas raciales, sociales, de origen migratoria, de edad y género, etc., de las fábricas de desigualdades son, por lo menos en muchos contextos urbanos, relativamente inextricables. El "motor racial de la desigualdad" no puede tomarse de manera aislada de los factores ya mencionados, pero tampoco es un resultado marginal, ya que como lo muestra el artículo de Barbary, Ramírez y Urrea pueden observarse efectos discriminatorios raciales en el caso de la clase media negra en la ciudad de Cali; y de acuerdo con Cunin en su análisis de los juegos de identificación socio-racial en Cartagena, aparece claramente un orden jerárquico de dominación/subordinación.

Los artículos de Agier y Quintín, Hoffmann y Barbary, Ramírez y Urrea tienen su origen en un mismo proyecto de cooperación entre el CIDSE y el IRD con el apoyo de COLCIENCIAS, "Movilidad, urbanización e identidades de las poblaciones afrocolombianas en la región del Pacífico colombiano sur" (título resumido), ${ }^{1}$ que desde 1996 hasta la fecha ha generado una serie de resultados novedosos y estudios cuantitativos y cualitativos ya publicados en forma de artículos y libros. A partir de estos resultados, se elaboran actualmente nuevos avances y trabajos de síntesis, de los 
cuales forman parte los tres artículos que aquí son publicados. ${ }^{2} \mathrm{El}$ artículo de Cunin sobre la ciudad de Cartagena, forma parte de su contribución al capítulo "etnicidad y ciudad" de un libro sobre las dinámicas urbanas en Colombia que redacta actualmente un equipo de investigadores de varias instituciones francesas. El artículo de Wade, ya conocido como un investigador pionero sobre la población negra colombiana desde el inicio de los años ochenta, constituye uno de sus más recientes trabajos en la dirección analítica aquí esbozada.

Los cinco artículos tienen diferentes escenarios geográficos rurales y urbanos de la población negra en Colombia. Los de Agier y Quintín, Hoffmann, y Barbary, Ramírez y Urrea se focalizan en el eje Cali-Costa Pacífica sur, aunque en sus análisis de los procesos políticos y sociales incluyen resultados válidos para la Costa Pacífica en su conjunto. Al respecto ver Mapas 1 (Zonas de expansão histórica da população negra na Colômbia) y 2 (Os quatro departamentos da Região Pacífica). Agier y Quintín combinan en su análisis de los procesos políticos identitarios resultados del trabajo de campo en el municipio de Tumaco, en la ciudad de Cali y en el Norte del Cauca. Mientras Hoffmann observa en detalle los procesos sociales en la sociedad rural-urbana del Pacífico sur, en el municipio de Tumaco; Barbary, Ramírez y Urrea analizan la geografía socio-racial de Cali (ver Mapa 3: Santiago de Cali por barrios, zonas pesquisadas), pero también avanzan en un ejercicio comparativo sobre la producción de identidades en espacios rurales y urbanos muy diferentes (Departamento del Chocó y la ciudad de Cali). El de Cunin se centra sobre Cartagena, ciudad de la Costa Caribe, y en uno de los barrios simbólicos de alta concentración de población negra (Chambacú); y el de Wade hace múltiples referencias empíricas a Barranquilla, la ciudad de mayor tamaño de la Costa Caribe, como espacio privilegiado de la producción cultural musical "costeña", pero también a la ciudad de Cali en sus anotaciones del rap y el grupo afrocolombiano Ashanty.

M. Agier y P. Quintín proporcionan un artículo que ofrece un marco general, apoyándose siempre en ejemplos concretos, del contexto sociopolítico de construcción de las identidades étnicas y territoriales y de las nuevas producciones culturales en las cuales operan las dinámicas de estructuración de las identidades sociales, culturales y políticas de las poblaciones negras y mulatas en Colombia. Lo interesante en la descripción y el análisis es que logran mostrar cómo el campo cultural hace referencia directa a la dimensión política identitaria, además de configurar una práctica de vida 
La población negra en la Colombia de hoy: dinámicas sociodemográficas...

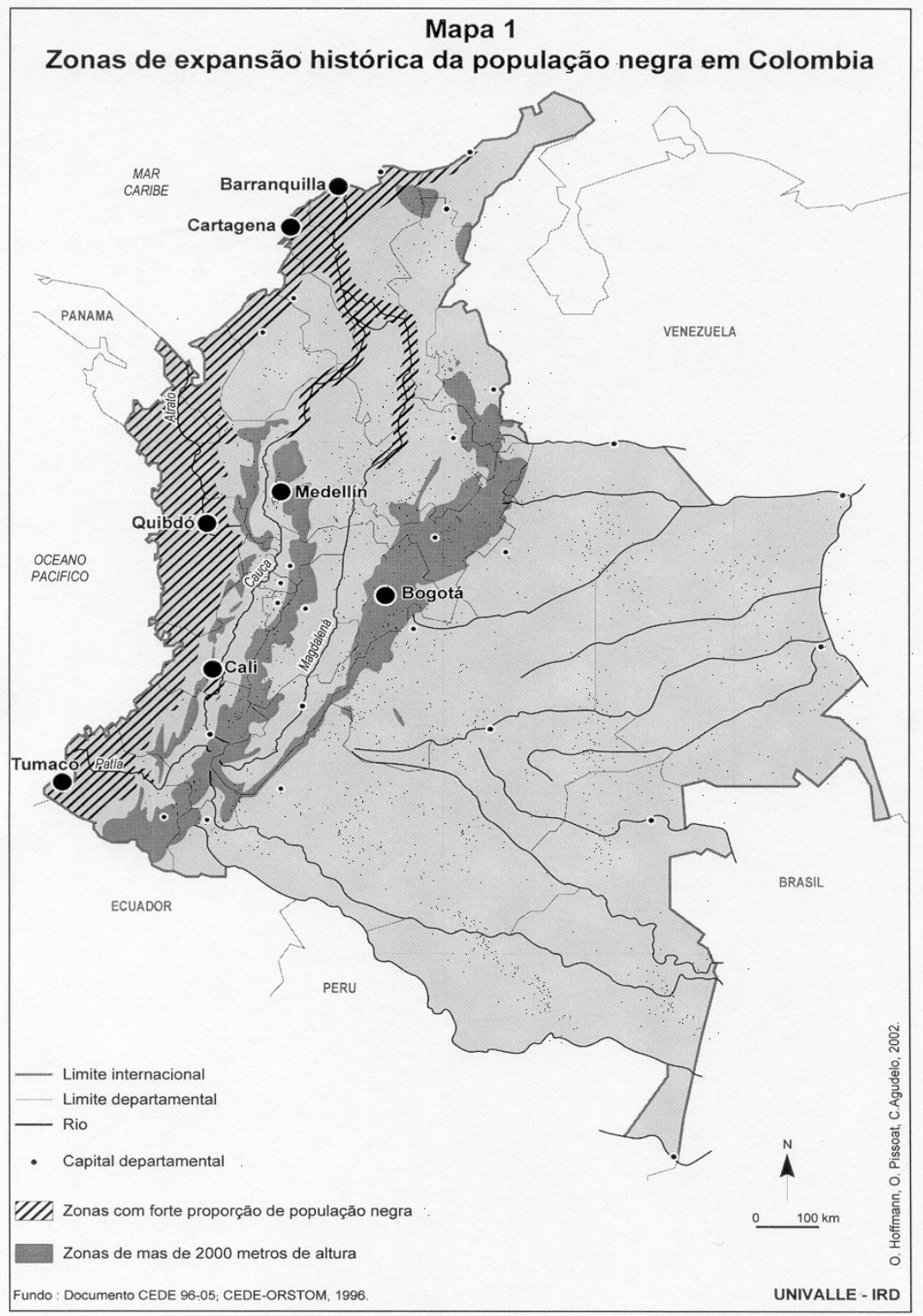


Olivier Barbary e Fernando Urrea

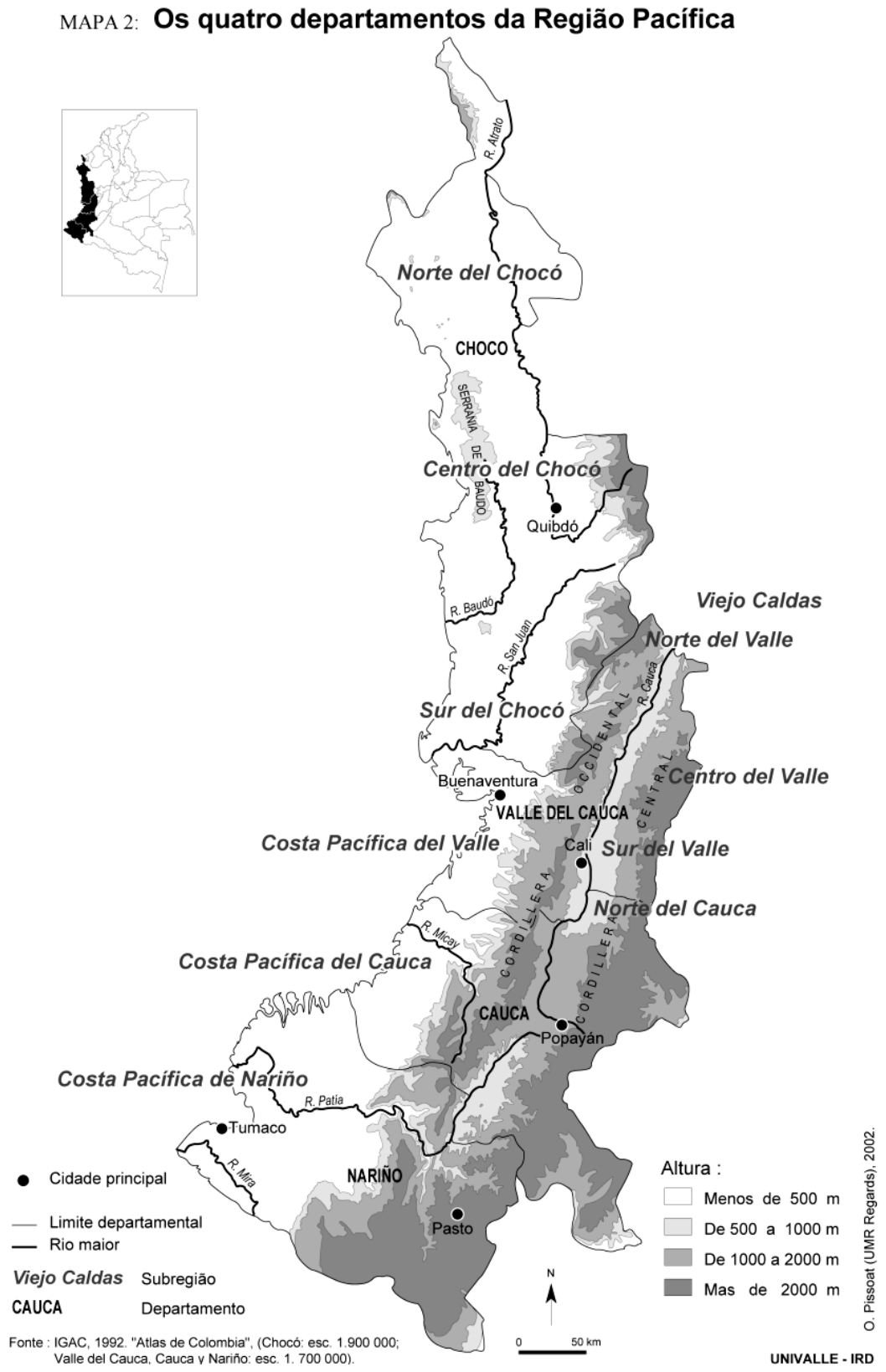


La población negra en la Colombia de hoy: dinámicas sociodemográficas...

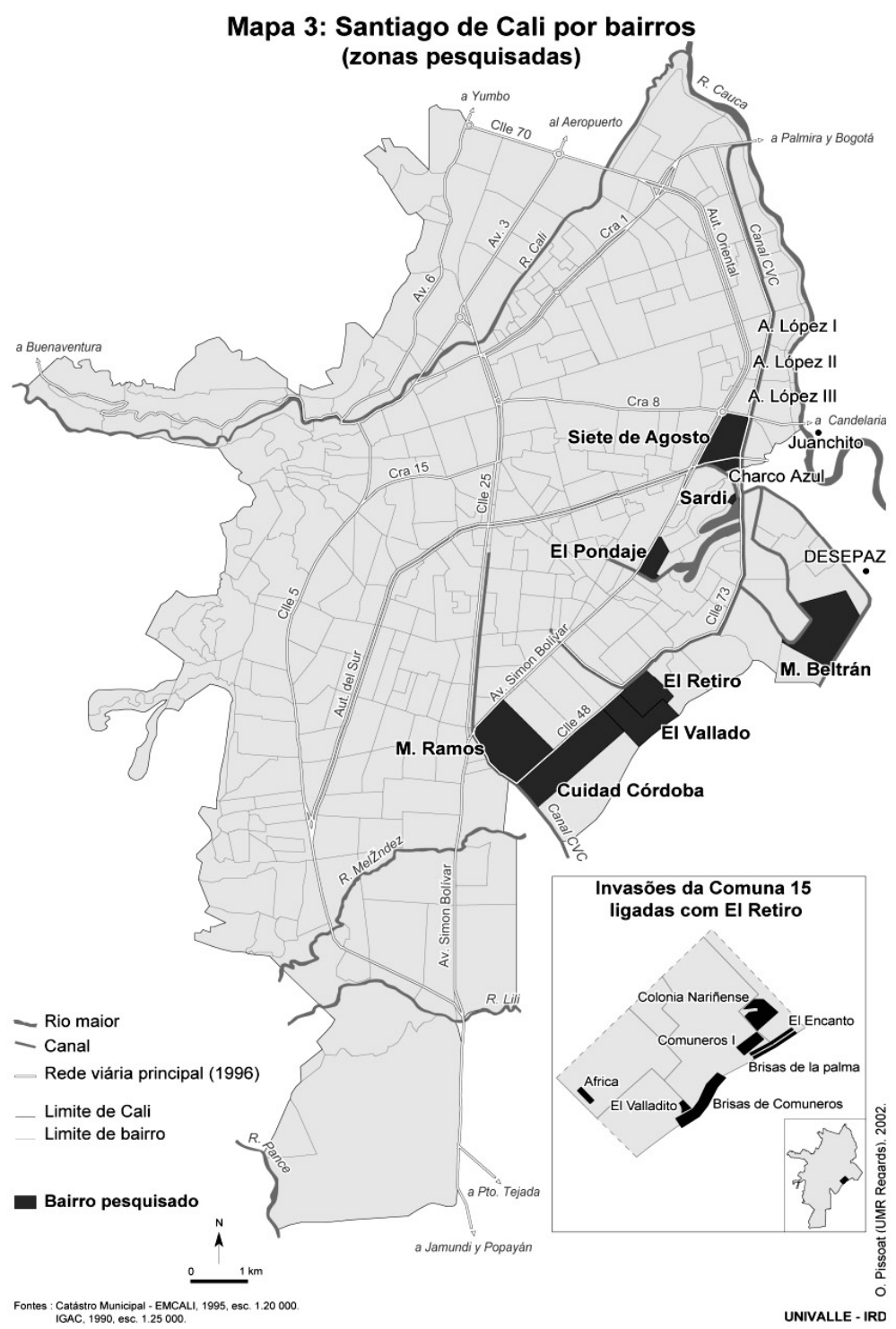


con implicaciones económicas en la sobrevivencia de los participantes activos. Los autores indagan así, cómo se ha construido la alteridad en los juegos de identidades de los afrocolombianos en los últimos años, aludiendo, a lo largo del artículo, a muchos problemas y resultados que se presentarán de manera más detallada en los tres artículos siguientes.

El texto de Agier y Quintín articula de un modo novedoso la temática de percepción de sí (y por lo mismo, del otro) con el fenómeno contemporáneo de la identidad cultural y su construcción a través de diferentes facetas a partir de la década del noventa en Colombia: desde la cultura como "mercancía" o inversión capitalista y profesional, pasando por el llamado trabajo de la memoria y el trabajo simbólico que aparece en las innovaciones culturales, hasta la producción de “imágenes” sobre la cultura del Pacífico y su reinvención en la ciudad de Cali. Los autores así apuntan a interpretar los nuevos fenómenos culturales "étnicos" de las poblaciones afrocolombianas en una perspectiva urbano-regional, entre el Pacífico y Cali, pero a la vez en el contexto de las transformaciones vividas por la sociedad colombiana en las últimas dos décadas. Finalmente, colocan una reflexión interesante y necesaria desde una dimensión ética-política sobre el papel de los intelectuales y el rol de los investigadores en los procesos de conciencia identitaria de corte "étnico-racial".

O. Hoffmann, a partir de un estudio de caso local (Tumaco, Nariño), presenta tres modelos socio-espaciales de organización económica y territorial de la región del litoral Pacifico. De manera analítica, estas tres configuraciones son separadas pero sin que ello signifique darles el sentido normalizado de tres "etapas de desarrollo" que se sucederían en el tiempo, sino más bien, de patrones de análisis que fungen, y eventualmente coexisten, en distintos contextos históricos y políticos, y a diferentes escalas local, regional y nacional. A través de la conjugación de estos diferentes tiempos y espacios, el artículo logra identificar el papel que distintos actores sociales, económicos y políticos juegan en el proceso de transformación acelerada que afecta este territorio y la población en la que en él reside. En el primer modelo, según las disposiciones de la nueva carta magna de 1993, las "comunidades negras" son entendidas como etnia, noción que se fundamenta en el argumento de la "especificidad" agraria y étnica de los habitantes del Pacífico; es decir en la existencia de una configuración socio-espacial muy peculiar, calificada como "dispositivo fluvial ribereño 'tradicional". El segundo dispositivo -el de la "modernización”-, hace 
énfasis, al contrario, sobre la indiferenciación de las sociedades locales, insertas, en mayor o menor grado, en procesos globales de articulación a las dinámicas nacional e internacional. Finalmente, el dispositivo de la "movilización político-étnica" corresponde a la emergencia reciente de configuraciones nuevas, resultados del cruce de los dos primeros modelos e integrando múltiples elementos nuevos.

Siguiendo el hilo de la dialéctica social, espacial y política entre los tres modelos, en la perspectiva de desparticularizar el $\mathrm{Pa}-$ cífico y rechazando las determinaciones ligadas a una supuesta "especificidad cultural" negra, la autora formula la esperanza de que este proceso desemboque en una oportunidad que siempre le ha sido negada al Pacifico: la construcción de una sociedad regional. Un tal proyecto, dado sus antecedentes históricos y las dinámicas sociales, económicas y políticas que se producen en el contexto actual, no puede basarse sino en la complementariedad de los dos "pilares" de estas dinámicas -los territorios negros y el sector agroindustrial- $y$, por lo tanto, en soluciones negociadas entre ambos. Desafortunadamente, las evoluciones recientes tienden más bien a la generalización y agravación de los conflictos sociales, económicos y políticos en la región, lo cual compromete fuertemente la probabilidad de una salida favorable. Es importante subrayar que lo que se juega en torno a esta problemática - aparentemente regional - desborda ampliamente las fronteras 'naturales' de las regiones costeras, en particular en dirección de Cali y otras grandes ciudades del país, en donde ahora se concentra gran proporción de la población afrocolombiana. Por esta razón, en cuanto espacio histórico y simbólico de origen de esta población, el Pacifico y su porvenir no pueden dejar de ocupar un lugar central en el debate político de la "cuestión negra" en Colombia.

Sin embargo, los hallazgos de O. Hoffmann para el Pacífico, no impiden que exista en la actualidad una dinámica de diferenciación fuerte entre los procesos de construcción de las identidades sociales, culturales y políticas de las poblaciones negras en los diferentes contextos urbanos del país; así lo muestran los dos artículos siguientes para los casos de Cali y Cartagena. El caso de Cali, como la primera ciudad de la región del Pacífico y con el mayor volumen absoluto de población negra en todo el país; y Cartagena, la segunda ciudad en importancia de la Costa Caribe colombiana, con el mayor peso relativo de gente negra entre las 15 principales ciudades colombianas. 
El artículo de O. Barbary, H. F. Ramírez y F. Urrea pone en evidencia la importancia del factor migratorio y de la urbanización masiva de la población afrocolombiana, en las dinámicas de construcción de nuevas "identidades étnicas" y de reivindicación ciudadana, y en su diferenciación según los contextos locales y regionales. Aprovechando dos tipos de fuentes estadísticas -el censo de 1993, para la región del Pacífico, y una encuesta demográfica llevada a cabo en Cali en 1998- las cuales son sometidas al análisis por medio de regresiones logísticas, así como datos cualitativos socioantropológicos, los autores realizan una interpretación sociológica de las determinaciones recíprocas entre el contexto territorial, económico y social en el cual se encuentra esta población, y los niveles y modalidades de la autoafirmación de una "identidad afrocolombiana". La conclusión principal es que las formas de afirmación de identidad son radicalmente diferentes para la población de la región pacífica y la población de Cali. Se trata en el primer caso de una reivindicación étnico territorial, mientras en el segundo, lo que está en juego es el acceso en igualdad de oportunidades a los mercados residenciales y laborales, y la denuncia de la discriminación socioracial. A la luz de estos resultados, el problema de la adecuación del marco jurídico de la Ley 70 con las necesidades y aspiraciones a la integración ciudadana de la población afrocolombiana, se coloca en nuevos términos, particularmente en la sociedad urbana.

Para los autores, los hallazgos cuantitativos y cualitativos sobre la percepción de la discriminación racial en Cali, permiten afirmar que ella es generalizada en diferentes espacios urbanos: mercado de trabajo, transporte público, arbitrariedad y maltrato policial, desiguales oportunidades escolares y en el acceso a servicios de salud, recreación, etc. Sin embargo, muestran que hay marcadas diferencias en esas percepciones de acuerdo a una serie de características de la población negra; en ese sentido resaltan la heterogeneidad sociodemográfica y socioeconómica de la misma. Por ello, factores tales como el origen geográfico de los migrantes, el nivel de escolaridad y la posición socio-ocupacional, así como la edad y el género son determinantes en la percepción de la discriminación racial y las modalidades de ella. En síntesis, se trata de una combinación de factores de clase social con origen socio geográfico, grupos etáreos y género.

E. Cunin propone en su artículo un análisis de los procesos de identificación socio-espacial de la ciudad de Cartagena, a través del estudio de las calificaciones étnico-racial que designan, en tér- 
minos émicos, los espacios y los habitantes de esta cuidad. Su análisis es un estudio de los mecanismos de la producción recíproca de territorios e identidades urbanas, en un contexto de alteridad según el color de piel, y en medio de aspiraciones a la ciudadanía y la urbanidad. Cunin analiza a lo largo de su artículo tres problemáticas específicas en esta ciudad: la significación de la imagen de "ciudad mestiza" a la que tradicionalmente ha sido asociada Cartagena; las interpretaciones diversas relacionadas con la erradicación de Chambacú -el barrio "negro" tradicional de la ciudad- hacia comienzos de los años setenta, barrio situado en la entrada de la ciudad histórica y turística; y las formas de apropiación territorial de los habitantes que reclaman una pertenencia étnica, dentro del contexto del multiculturalismo de los años noventa.

La autora muestra cómo la gestión cotidiana de la diferencia contribuye a la producción de espacios urbanos, en un movimiento de ida y vuelta entre identificación y territorialización. Se trata de una dinámica compleja de confrontación y coexistencia sobre un mismo territorio urbano, tomando en cuenta los mecanismos de identificación del yo y del otro en el juego de las relaciones interraciales. Por otra parte, más que un fracaso del multiculturalismo en Cartagena, Cunin interpreta su débil desarrollo como una consecuencia de la imposición de procesos de identificación situacionales e interaccionales en la perspectiva goffmaniana, a través de los cuales los habitantes de Cartagena construyen al mismo tiempo su derecho a la urbanidad y a la diferencia.

El artículo de P. Wade, el último de este dossier, propone una contextualización desde la perspectiva de la antropología política de la construcción de una identidad "africana" y "negra" en las prácticas culturales que se desarrollan a través de las músicas populares colombianas de la Costa del Caribe, desde los años 50 y 60 (sobre todo en el porro y la cumbia) hasta los años 90 con la presencia de otras músicas, regiones e influencias (el currulao del Pacífico, la champeta en Cartagena, el rap en los grupos de jóvenes negros en Cali). La "africanidad" y la "negritud" no son vistas en términos de herencia o continuidad en las prácticas culturales concretas de generaciones sucesivas, si bien el autor no desconoce el aporte histórico de la tradición africana; por el contrario, son nociones descritas y analizadas en cuanto representaciones elaboradas a partir de los contextos sociales, culturales o políticos en los que han vivido y participado los actores de la producción musical: músicos, públicos, críticos, etc. Por ello, este punto de vista conduce al autor a insistir sobre la forma dinámica en que las identida- 
des individuales y colectivas se producen en referencia a múltiples elementos de una rica "herencia cultural" europea, africana e indígena, los cuales toman cuerpo cada uno en grados diversos y cambiantes en las prácticas culturales de los individuos y los grupos. En tal sentido se produce una encarnación cultural de lo negro, lo blanco y lo indígena a través de esos múltiples elementos y variaciones.

El estudio de Wade se inscribe en sus últimos trabajos dedicados a las relaciones interraciales en Colombia a través de la producción musical, en particular su libro al respecto, ${ }^{3}$ en el cual realiza el estudio de la construcción de las identidades nacional, regionales y raciales, generadas históricamente en Colombia a lo largo del siglo XX, a través de la música popular y el baile "costeños" (de la Costa Caribe colombiana). La perspectiva analítica de Wade en este terreno específico de las prácticas culturales de la música y el baile como producción social, en marcada en una antropología política que analiza la alteridad de las relaciones interraciales, comparte también supuestos centrales con los otros artículos: los de una mirada crítica histórica y no esencialista, de las dimensiones de raza, clase social, género y origen regional. Es un orden social jerarquizado racialmente, que al mismo tiempo ofrece una diversidad regional en las relaciones interraciales.

Los cinco artículos trazan en tres dimensiones -sociodemográfica, sociopolítica y sociocultural- la construcción de las identidades socio-raciales en la Colombia contemporánea a partir de los contextos históricos específicos, nacional, regional y local. Son identidades resultado del orden social racializado; por lo que el fenómeno del racismo afecta considerablemente las distintas categorías de clases sociales en una lógica recíproca de "blaqueamiento" versus "ennegrecimiento" de los fenotipos. Sin existir una relación mecánica, las capas altas de la población tienden a ser asociadas al "blanco" mientras los sectores populares al "negro" o el "indio". Hay fuertes variaciones regionales pero esta relación es generalizable a todo el país.

En este sentido los artículos de este dossier se centran por una parte en la temática del racismo y su percepción por diferentes agentes implicados, ya sea como subordinados o en calidad de grupo no subordinado racial y socialmente, ya sea en Cali, el Pacífico o Cartagena. Por otra, en la dinámica de los movimientos sociales negros y las prácticas culturales en el Pacífico, en Cali, o en la Costa del Caribe, y en este último caso, su incidencia e interacción con la sociedad dominante del interior del país a lo largo del siglo XX. 
Los estudios aquí presentados tienen una perspectiva analítica común respecto a la "etnicidad" en las sociedades contemporáneas, evitando las clasificaciones esencialistas. Por lo mismo, resaltan la dinámica histórica de un orden social racializado y las relaciones interraciales de dominación y subordinación que él impone en los contextos sociales y locales concretos.

Los autores del dossier son de diferentes disciplinas: antropología, sociología y estadística, con aportes que se apoyan en metodologías cuantitativas y cualitativas. Sin embargo, los resultados muestran hallazgos coincidentes en unos casos, en otros complementarios; sobre todo esto es válido si comparamos los artículos de Cunin y Wade, investigadores independientes, con los tres artículos de Agier y Quintín; Hoffmann; y Barbary, Ramírez y Urrea, los cuales han formado parte de un mismo programa de investigación, como se advirtió antes. Sin pretender entregar materiales que representen el conjunto de las tendencias de los estudios sobre poblaciones negras en Colombia, este número de la revista, por el contrario, ha querido privilegiar los resultados de investigaciones recientes que abren nuevas direcciones socioantropológicas y sociodemográficas a los estudios de etnicidad y raza en América Latina, sin perder el horizonte de los temas sensibles relacionados con el racismo y su interacción con las diversas formas de desigualdad social en los países latinoamericanos.

\section{Notas}

1. El título original del proyecto CIDSE-IRD es: "Organización social, dinámicas culturales e identidades de las poblaciones afrocolombianas del pacífico y suroccidente en un contexto de movilidad y urbanización”.

2. El equipo de investigación CIDSE (Centro de Documentación e Investigación Socioeconómica, de la Facultad de Ciencias Sociales y Económicas de la Universidad del Valle, Cali) - IRD (Institut de Recherche pour le Développement, France) es detallado más adelante en los artículos de O. Hoffmann, y de O. Barbary, H.F. Ramírez y F. Urrea. COLCIENCIAS es el Instituto para el Desarrollo de la Ciencia y Tecnología de Colombia, "Francisco José de Caldas". Además de los autores de los tres artículos este proyecto reunió a otros cinco investigadores colombianos y dos franceses; uno de ellos, Olivier Pissoat, geógrafo, autor de la mayor parte de los mapas sobre el Pacífico y la ciudad de Cali y coautor con O. Hoffmann de algunos artículos.

3. Music, "race" and nation: música tropical in Colombia, Chicago, University of Chicago Press, 2000. 